This is a preprint of the article: Foerster, R. M. (2018). “Looking-at-nothing” during sequential actions: Longterm memory-based eye scanning of remembered target locations. Vision Research, 144, 29-37. doi.org/10.1016/j.visres.2018.01.005. The article has been published in its final form at https://doi.org/10.1016/j.visres.2018.01.005. @ 2018. This manuscript version is made available under the CCBY-NC-ND 4.0 license http://creativecommons.org/licenses/by-nc-nd/4.0/

\title{
“Looking-at-nothing” during sequential sensorimotor actions: Long-term memory-based eye scanning of remembered target locations
}

\author{
Rebecca M. Foerster ${ }^{1,2}$ \\ ${ }^{1}$ Department of Psychology and ${ }^{2}$ Cluster of Excellence Cognitive Interaction Technology, \\ Bielefeld University, Germany
}

16

(1)

(8)

Correspondence: Rebecca M. Foerster, Neuro-cognitive Psychology, Department of Psychology, Bielefeld University, P.O. Box 100131, D-33501 Bielefeld, Germany, +49521/1064503, rebecca.foerster@uni-bielefeld.de 
1 Abstract

2 Before acting humans saccade to a target object to extract relevant visual information. Even

3 when acting on remembered objects, locations previously occupied by relevant objects are

4 fixated during imagery and memory tasks - a phenomenon called "looking-at-nothing”. While

5 looking-at-nothing was robustly found in tasks encouraging declarative memory built-up,

6 results are mixed in the case of procedural sensorimotor tasks. Eye-guidance to manual targets

7 in complete darkness was observed in a task practiced for days beforehand, while investigations using only a single session did not find fixations to remembered action targets. Here, it is asked whether looking-at-nothing can be found in a single sensorimotor session and thus independent from sleep consolidation, and how it progresses when visual information is repeatedly unavailable. Eye movements were investigated in a computerized version of the trail making test. Participants clicked on numbered circles in ascending sequence. Fifty trials were performed with the same spatial arrangement of 9 visual targets to enable long-term memory consolidation. During 50 consecutive trials, participants had to click the remembered target sequence on an empty screen. Participants scanned the visual targets and also the empty target locations sequentially with their eyes, however, the latter less precise than the former. Over the course of the memory trials, manual and oculomotor sequential target scanning became more similar to the visual trials. Results argue for robust looking-at-nothing during procedural sensorimotor tasks provided that long-term memory information is sufficient.

Keywords: looking-at-nothing, visual attention, eye movements, action control, long-term memory, trail making test 


\section{Introduction}

When interacting with our environment, we use our eyes to extract task-relevant sensory visual information of target objects. However, the eyes are sometimes shifted to remembered targets, although visual information is not available - a phenomenon called looking-at-nothing (Ferreira, Apel, \& Henderson, 2008; Johansson, Holsanova, Dewhurst, \& Holmqvist, 2011; Richardson, Altmann, Spivey, \& Hoover, 2009; Richardson \& Spivey, 2000). Looking-at-nothing refers to the behavior of saccading to empty locations, e.g., on a screen or behind an occluder where task-related material had been available or is expected to be. This behavior is robustly observed during visual imagery and memory-recall tasks (Brandt \& Stark, 1997; Johansson et al., 2011; Johansson, Holsanova, \& Holmqvist, 2005, 2006; Johansson \& Johansson, 2013; Laeng \& Teodorescu, 2002; Mast \& Kosslyn, 2002; Noton \& Stark, 1971a, 1971b; Spivey \& Geng, 2001). It has been hypothesized that saccading to locations that have previously been occupied by to-be-remembered material might facilitate memory encoding and recall (Johansson et al., 2011; Johansson \& Johansson, 2013; Laeng \& Teodorescu, 2002). As a covert shift of attention obligatorily precedes every gaze shift (Deubel \& Schneider, 1996), the facilitated recall might be grounded on attention allocation to the location previously occupied by the to-be-recalled object. In any case, the phenomenon of looking-at-nothing proves that humans can use memory information to direct their gaze to task-relevant locations in space.

Imagery and memory-recall tasks encourage explicit or declarative memory encoding, because memory retrieval is explicitly required to solve the task. Therefore, the question arises whether the phenomenon of looking-at-nothing can also be found in tasks that are dominated by implicit procedural memory such as sensorimotor tasks. Fixations to actiontarget locations in the absence of visual information were indeed found in a well-practiced cup-stacking task (Foerster, Carbone, Koesling, \& Schneider, 2012). Participants performed the 14-days trained cup-stacking sequence (speed stacking or sport stacking, see also Foerster 
et al., 2011) first with normal lighting and afterwards in complete darkness, while gaze was recorded. Even without any visual information, participants saccaded to upcoming hand-target locations slightly before the hands reached the locations. The eye-hand dynamics as well as the sequence of fixated locations, the scanpath, were highly similar between light and dark condition. The results argue that procedural long-term memory (LTM) can indeed be used to direct attention and gaze to target locations when visual information is not available.

Contrastingly, Flanagan et al. (2008) found only a loose relationship between eye and hand movements across visual conditions in two sensorimotor tasks. In one experiment, participants pointed to visual versus remembered targets. In another experiment, participants manipulated visual objects or manipulated objects behind a shutter in complete darkness. Both tasks were not practiced beforehand. Pointing target locations were randomly chosen prior to each trial and participants reacted to a specific configuration only once before they had to point from memory, both times in a self-regulated sequence. Similarly, each objectmanipulation sequence was repeated only four times with visual information before participants had to act from memory. Thus, procedural memory might have been too fragile to trigger looking-at-nothing in these single-session investigations in which the required motor actions were not practiced much. As it is known that especially procedural learning strongly benefits from sleep consolidation, it is also possible that looking-at-nothing during sensorimotor procedures occurs robustly only after sleep consolidation (Stickgold, 2005; Walker, Brakefield, Morgan, Hobson, \& Stickgold, 2002). Not only manual but also the corresponding oculomotor trajectory might have been sufficiently consolidated for looking-atnothing behavior to occur only in multiple day investigations.

Here, it is investigated whether looking-at-nothing can robustly be found in a sensorimotor task within a single session, i.e., without intermittent sleep consolidation of the sensorimotor procedure. Crucially, participants started with a longer series of trials with visual information available, allowing to building up procedural memory of a sufficient 
strength. Afterwards, gaze behavior was investigated over an equally long series of trials without visual information available. Thereby, it can be revealed how robustly participants scan remembered action-target locations with their eyes from LTM when short-term memory has already faded. In this case, looking-at-nothing might be abandoned, modified or intensified provided that action-completion feedback is still available.

Eye movements were recorded while participants performed a computerized version of the number connection test or trail making test A (Army Individual Test Battery, 1944; Foerster \& Schneider, 2015; Reitan, 1958). In this sequential sensorimotor task, participants had to click as fast as possible on numbered circles in ascending order (here 1 -9). Visual and procedural LTM encoding was enabled by a 50-trials visual action phase with the same spatial arrangement of nine visual targets. In a consecutive 50-trials memory-based action phase, participants were asked to click at the remembered locations on a blank screen in the same sequence as during the visual phase. Auditory feedback signaled clicking success throughout the experiment. Scanpath analyses were performed to reveal how precisely participants scanned the visual and remembered target locations in sequence over the course of the visual and memory-based action phases.

\section{Methods}

\subsection{Participants}

Eleven right-handed students (4 male and 7 female) from Bielefeld University, Germany, participated in the experiment. Participants’ mean age was 25 years. All participants had either normal or corrected-to-normal visual acuity. All were naïve with respect to the purpose of the study, gave informed consent, and were paid for their participation. The study was conducted in accordance with the Code of Ethics of the World Medical Association (Declaration of Helsinki). 


\subsection{Apparatus and Stimuli}

The experiment was controlled by the Experiment Builder software (SR Research, Ontario, Canada) on a Dell Optiplex 755 computer. The stimuli were displayed on a 19-in. CRT monitor (ViewSonic Graphics Series G90fB) with a refresh rate of $100 \mathrm{~Hz}$ and a resolution of 1,024 x 768 pixels controlled by an ATI Radeon HD 2400 Pro graphics card. The computer mouse and keyboard as well as an extra-large mouse pad (88 x $32 \mathrm{~cm})$ were used. Each participant's right gaze position was recorded by an EyeLink 1000 tower system (SR Research). The eye tracker’s sampling rate was 1,000 Hz, and participants’ viewing distance was fixed at $71 \mathrm{~cm}$ with a chin and forehead rest throughout the experiment. The cursor position was recorded with the monitor's sampling rate of $100 \mathrm{~Hz}$. Color and luminance were measured at the screen center in CIE Lxy coordinates using an X-Rite i1 Pro spectrophotometer.

All stimuli were black $\left(\mathrm{L}=0.3 \mathrm{~cd} / \mathrm{m}^{2}, \mathrm{x}=.32, \mathrm{y}=.33\right)$ and displayed on a gray background $\left(\mathrm{L}=78.9 \mathrm{~cd} / \mathrm{m}^{2}, \mathrm{x}=.29, \mathrm{y}=.30\right)$. The mouse cursor was an upwards pointing arrow of approximately .68 degrees of visual angle $\left({ }^{\circ} \mathrm{v} . \mathrm{a}.\right)$ width and $1.69^{\circ} \mathrm{v} . \mathrm{a}$. height. The target stimuli consisted of 9 numbers (Arial, font style bold, font size 35), each surrounded by an unfilled circle $\left(2.04^{\circ} \mathrm{v}\right.$.a. diameter, line width 6). The first circle was located in the center of the screen. The spatial distribution of the other 8 circles was randomly generated with the prerequisite that each outer field of an imagined 3 by 3 grid contained one circle and circles had a minimal distance of $2.04^{\circ} \mathrm{V}$.a. to each other (from border to border) as well as to the screen border. The minimal distance between the nearest two circles 5 and 7 happened to be $5.93^{\circ} \mathrm{v} . \mathrm{a}$. in the generated display (from center to center). The same spatial configuration was used throughout the entire experiment (Figure 1). 


\subsection{Procedure}

The experiment was divided into a first visual action phase with numbered circles on the screen and a consecutive memory-based action phase with a blank screen. Each phase started with a written instruction on the screen followed by a 9-point calibration and validation procedure. Only calibrations with averaged validation accuracy below $1.0^{\circ} \mathrm{v}$.a. were accepted. In the visual phase, participants had to click as fast as possible in ascending order on the 9 numbered circles presented on the screen (Figure 1, top). Participants were informed that the configuration of target stimuli stayed the same throughout the whole experiment. An example trial preceded the visual phase. In the subsequent memory phase, only the mouse cursor was displayed on the grey background (Figure 1, bottom), and participants were instructed to click as fast as possible on the locations that were previously occupied by the numbered circles in the same sequence as before. A click was counted as correct, if the mouse cursor was within a diameter of $3.06^{\circ} \mathrm{v}$.a. around the current target's center. A correct click was followed by a high-pitched tone. An incorrect click was followed by a low-pitched tone. After all 9 circles were clicked on in the right order trial completion time was displayed on the screen. Each trial was preceded by a central fixation on a black ring $\left(.45^{\circ}\right.$ v.a. outer size and $.11^{\circ}$ v.a. inner size). The visual phase as well as the consecutive memory phase consisted of 5 blocks à 10 trials, adding up to 100 trials in total. A block information display separated each block. Participants could start each block and trial by pressing the space bar. Participants were allowed to take self-paced breaks in-between blocks and trials. All participants completed the experiment within 40 minutes. The participant with the fastest time in each phase earned $6 €$ extra. 


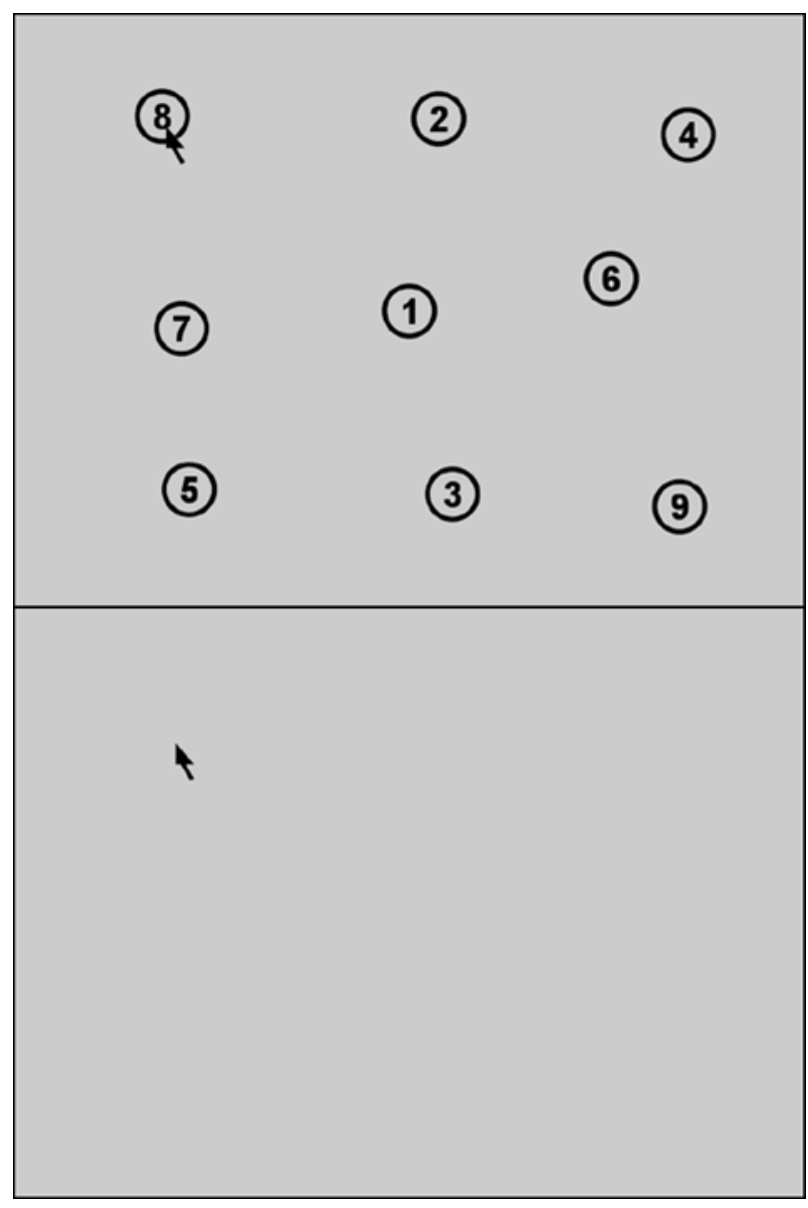

Figure 1. Displays during sequential clicking in the visual (top) and memory (bottom) phase of the experiment.

5

6

7

\subsection{Analysis}

The SR Research Data Viewer software’s implemented algorithm was used to detect fixations, saccades, and blinks (velocity threshold of $30^{\circ} \mathrm{v} . \mathrm{a} . / \mathrm{s}$ and acceleration threshold of $8,000^{\circ}$ v.a. $\left./ \mathrm{s}^{2}\right)$. The following variables were analyzed as dependent variables: Trial completion time, number of clicking errors, number of fixations, distance of fixations and clicks to the current target, eye-hand spans, and scanpath similarity. The eye-hand time span refers to the time interval between a fixation at a specific target and the corresponding action - in this case a click - to the target (cf. Butsch, 1920; Furneaux \& Land, 1999; Sailer, Eggert, Ditterich, \& Straube, 2000). The eye-hand unit span refers to the number of actions completed in between a fixation at a specific target and the corresponding action - in this case a click to the target (cf. Foerster et al., 2011, 2012). The eye-hand target distance span indicates 
whether the eye or the cursor is nearer to the current target. It is calculated by subtracting for each $100 \mathrm{~Hz}$ sample the distance of the eye sample position (down-sampled to $100 \mathrm{~Hz}$ by averaging the eye position) to the current target's center from the distance of the cursor position (measured with $100 \mathrm{~Hz}$ ) to the current target's center (cursor-target distance minus eye-target distance). Therefore, the eye-hand spans reveal how far ahead the eyes are with respect to the hand within an action sequence. Positive eye-hand spans indicate that the eye is anticipatorily guiding the manual action while negative eye-hand spans indicate that the eye is following the manual action. Scanpath comparisons were performed using FuncSim (Foerster \& Schneider, 2013a, 2013b) and MultiMatch (Dewhurst et al., 2012; Jarodzka, Holmqvist, \& Nyström, 2010). These methods will be explained in the Results section. Violations of sphericity were corrected using the Greenhouse-Geisser $\varepsilon$ (uncorrected degrees of freedom are reported to facilitate reading). A chance level of .05 was applied. Data processing and aggregation were performed using Matlab R2013b and Microsoft Excel 2010. Statistical analyses were conducted with IBM SPSS Statistics 22.

\section{Results}

The Result section is divided into three parts. In the first part, manual performance and gaze control during the last visual block (block 5) is compared to the first memory block (block 6). The second part provides an analysis of the development of manual and gaze measures when visual information is repeatedly not available. Third, it is investigated whether visually marked as well as remembered action target locations are scanned sequentially with the eyes.

\subsection{Performance and gaze patterns with visual versus remembered action targets}

To reveal the effects of the lack of visual information, click performance and gaze patterns of the last visual block (block 5) were compared to the first memory block (block 6). 
1 Trial completion time, number of clicking errors, number of fixations, and the distance of

2 fixations and clicks to the current target increased significantly as soon as visual information

3 was no longer available and the eye-hand unit span and the eye-hand target distance span

4 decreased significantly (Figure 2; time: $t(10)=3.82, S E=7.74, p<.01$; number of clicking

5 errors: $t(10)=3.04, S E=18.45, p<.05$; number of fixations: $t(10)=3.15, S E=17.28, p<.05$;

6 fixation distance: $t(10)=4.93, S E=.72, p<.001$; click distance: $t(10)=2.66, S E=.82, p<.05$; eye-

7 hand unit span: $t(10)=3.10, S E=.13, p<.05$; eye-hand target distance span: $t(10)=10.21$,

$8 S E=.15, p<.001)$. The eye-hand unit span decreased from positive to negative values, while

9 the eye-hand target distance span remained positive. The eye-hand time span was not significantly different between the visual and the memory phase $(t(10)=.99, S E=4.56, p=.34)$ due to a high amount of variability, although its average decreased from positive to negative values (Figure 2). Thus, performance dropped when visual information was no longer available and eye movements were less related to the action target locations. During the visual phase, fixations mainly landed at the current action target (eye-hand unit span of 0 ) or at positions becoming targets even later resulting in a slightly positive eye-hand unit span of .13. The amount of fixations landing at completed target locations increased when acting on remembered target locations, indicated by a slightly negative eye-hand unit span of -.32. Nevertheless, the eye was on average nearer to the current target than the cursor was (positive eye-hand target distance span), even though with a smaller distance between eye and cursor than during the last visual block. 


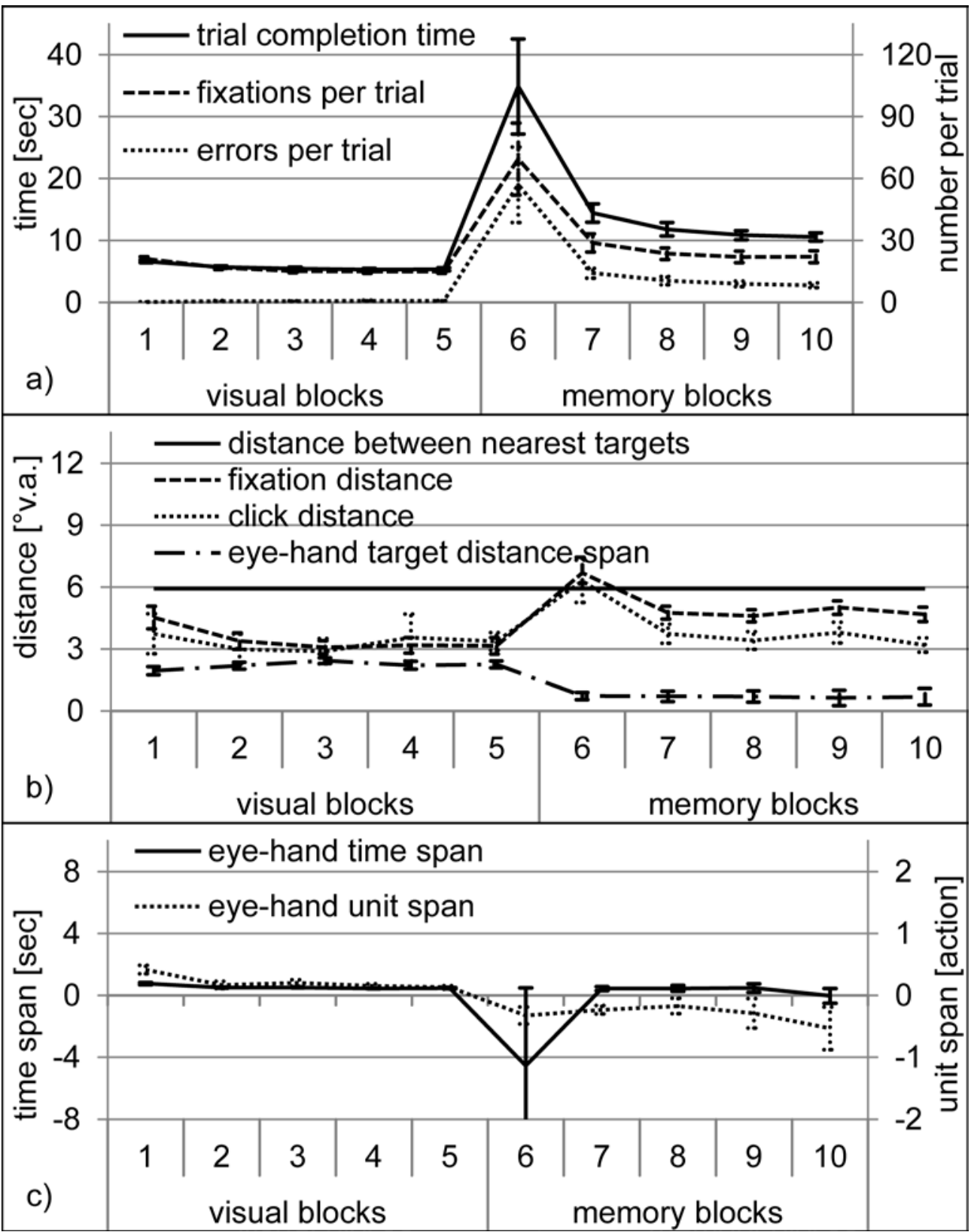

Figure 2. a) Trial completion times in seconds (solid line and left y-axis) as well as numbers of fixations (dashed line) and clicking errors (dotted line) in number per trial (right y-axis) across all blocks. b) Distance of fixations (dashed line) and clicks (dotted line) to the current target as well as the eye-hand target distance span (dotdashed) in ${ }^{\circ} \mathrm{v}$.a. across all block. The solid line displays the distance between the nearest two targets ( 5 and 7 ) which was $5.93^{\circ} \mathrm{v}$.a. c) Eye-hand time span (solid line and left y-axis) in milliseconds and eye-hand unit span (dotted line and right y-axis) in number of actions across all blocks. Error bars represent standard errors of the mean.

\subsection{Performance and gaze patterns when visual targets are repeatedly unavailable}

When visual information was repeatedly not available, performance and gaze values changed significantly in direction to the visual-phase values as indicated by significant block 
1 ANOVAs. (Figure 2; time: $F(4,40)=9.00, \varepsilon=.26, p<.05, \eta_{p}{ }^{2}=.47$, linear trend $p<.01$; number

2 of clicking errors: $F(4,40)=6.39, \varepsilon=.26, p<.05, \eta_{p}{ }^{2}=.39$, linear trend $p<.05$; number of

3 fixations: $F(4,40)=7.38, \varepsilon=.26, p<.05, \eta_{p}{ }^{2}=.43$, linear trend $p<.05$; fixation distance:

$4 \quad F(4,40)=6.86, \varepsilon=.40, p<.05, \eta_{p}^{2}=.41$, linear trend $p<.05$; click distance: $F(4,40)=7.37, \varepsilon=.37$,

$5 p<.05, \eta_{p}{ }^{2}=.42$, linear trend $p<.05$ ). In the first memory block (block 6 ), the distance of

6 fixations and clicks to the current target was not significantly different from the distance of

$7 \quad 5.93^{\circ} \mathrm{v}$.a. between the nearest two targets (fixation: $t(10)=1.00, S E=.43, p=.34$ and clicks:

$8 t(10)=.35, S E=.51, p=.73$ ). Already during the second memory block (block 7), fixations and

9 clicks landed significantly nearer to the current target than to any other target (fixation: $t(10)=3.75, S E=.25, p<.01$ and clicks: $t(10)=4.76, S E=.39, p<.001$, tested against the distance of $5.93^{\circ} \mathrm{v} . \mathrm{a}$. between the nearest two targets). Thus, clicking performance approached a normal level and eye movements were again related to the current action target locations when participants were familiarized with the memory-clicking task. However, the eye-hand unit span remained slightly negative throughout memory-based acting and did not change significantly over the course of the memory phase $\left(F(4,40)=.87, \varepsilon=.35, p=.40, \eta_{p}{ }^{2}=.08\right)$. Numerically, it even decreased further. Thus, the eye-hand unit span either remained its dissimilarity to the visual phase over the course of the memory phase. The eye-hand time span and the eye-hand target distance span did not change significantly over the course of the memory phase (eye-hand time span: $F(4,40)=.94, \varepsilon=.25, p=.36, \eta_{p}{ }^{2}=.09$; eye-hand target distance span: $\left.F(4,40)=.06, \varepsilon=.47, p=.94, \eta_{p}^{2}=.01\right)$.

\subsection{Sequential scanning of visual and remembered action target locations}

How similar did participants scan the target locations in sequence with and without seeing the targets on the screen? Scanpath similarity, i.e., the similarity of fixation location sequences was calculated using two multi-dimensional scanpath-similarity methods. The first 
method is MultiMatch (Dewhurst et al., 2012; Jarodzka et al., 2010), a vector-based method that calculates separate sequence similarity scores for the dimensions vector, location, duration, length, and direction. Scores are normalized between 0 (no similarity) and 1 (perfect similarity). The second method is FuncSim (Foerster \& Schneider, 2013a, 2013b), a functional sequencing method that is adapted to compute ocular scanpath similarity in sequential motor actions. It calculates difference scores for the dimensions location, duration, length, and direction by aligning the fixations within the compared paths based on the performed sub-actions. In a first step, fixations belonging to the same sub-action are averaged (method 'average' in Foerster \& Schneider, 2013b). In a second step, location, duration, length, and direction differences are calculated within corresponding sub-actions of two scanpaths. With respect to the clicking task, fixations performed after the click on the last target until the click on the current target belong to the same sub-action. In addition, FuncSim calculates scanpath difference scores for each dimension between one of the scanpaths and its scrambled derivative. These scores serve as random baseline difference scores. The scrambling procedure assigns the fixations performed during a sub-action to another randomly chosen sub-action. In this way, fixations are shuffled randomly around as if the click-action sequence would have been different. As the scrambled scanpath consists of actually observed fixations, its comparison to the scanpath in the correct order constitutes a highly conservative random baseline difference score. MultiMatch and FuncSim provide Matlab toolboxes (download links: http://wiki.humlab.lu.se/dokuwiki/doku.php?id=public:useful_links and http://www.uni-bielefeld.de/psychologie/abteilung/arbeitseinheiten/01/Research/FuncSim) which were used for the following analyses.

As scanpaths can only be compared pairwise, for each participant all 10 trials of the last visual block (block 5 as reference block) were individually compared to all 10 trials of each of the five memory blocks, resulting in 100 comparisons per block. For random baseline calculation, the last visual block (reference block 5) was used. Each of the 10 trials of the last 
1 visual block (block 5) was compared to 10 randomly-chosen sequence-scrambled derivatives,

2 resulting in 100 random baseline comparisons. The same scrambled scanpath comparisons were used for FuncSim and MultiMatch. Only the values of the dimension location are reported here, because the research question was whether visually marked and empty target locations were scanned in a similar sequence. Paired $t$-tests were used to test whether the scanpaths of the last visual block (block 5) were more similar to their own scrambled derivatives or to the memory-based scanpaths of blocks 6-10. The FuncSim location difference scores were additionally compared to the nearest distance of two target locations in the display $\left(5.93^{\circ}\right.$ v.a.) and to the click acceptance region (3.06 ${ }^{\circ}$ v.a.) via one-sample $t$-tests. Mean MultiMatch location sequence similarities between the last visual block (block 5) and the memory blocks 6-10 ranged from .89 to .92 indicating high scanpath similarities between the visual and the memory phase (Table 1 and Figure 3). These similarity scores across the visual and the memory phase were not significantly different from each other $\left(F(4,40)=.56, \varepsilon=.47, p=.57, \eta_{p}{ }^{2}=.47\right)$, indicating that the last visual block (block 5) was not more or less similar to specific memory blocks. Importantly, all location sequence similarities were significantly higher than the random baseline similarities (all $p s<.01$ ).

Mean FuncSim location sequence differences between the last visual block (block 5) and the memory blocks 6-10 ranged from 3.34 to $3.76^{\circ}$ v.a. indicating likewise high scanpath similarities between the visual and the memory phase (Table 1 and Figure 4). These difference scores across the visual and the memory phase were not significantly different from each other $\left(F(4,40)=2.29, \varepsilon=.54, p=.12, \eta_{p}^{2}=.19\right)$, indicating that the last visual block (block 5) was not or less similar to specific memory blocks. Importantly, all location sequence differences were significantly smaller than the random baseline differences (all $p s<.001$ ). Moreover, all blocks' location sequence differences were significantly smaller than the $5.93^{\circ} \mathrm{v} . \mathrm{a}$. distance between the nearest two circles (all $p s<.001$ ). Mean location sequence differences of the last visual block (block 5) to the memory blocks 7-10 were also not 
1 significantly different from the click-acceptance area of $3.06^{\circ} \mathrm{v} . \mathrm{a}$. around the targets. Thus,

2 fixations landed approximately within the same circle area for each sub-action of the manual

3 sequence whether visual targets were available or not. This sequential scanning of

4 remembered target locations was robust across the memory blocks. Similarity scores,

5 difference scores, and $t$-test values are summarized in Table 1. Exemplary scanpath pairs

6 along with their FuncSim and MultiMatch comparison scores can be seen in Figure 4 and

7 Figure S1. Corresponding slow motion eye and cursor movements (100 Hz) can be seen in the

8 Supplementary Material.

Table 1. Mean location sequence MultiMatch similarity and FuncSim difference values of the comparisons of all trials of the last visual block (block 5) to all trials of the memory blocks (blocks 6-10) as well as to 10 scrambled derivatives per block 5 trial (5s). Values are presented along with the paired $t$-test values for the performed scanpath similarity comparisons (all against 5:5s, FuncSim values against the distance of $5.93^{\circ} \mathrm{v}$.a. between the nearest two circles and against the click acceptance region of $3.06^{\circ} \mathrm{v}$.a.). Significance levels are marked by asterisks $\left({ }^{*} p<.05, \stackrel{* *}{p}<.01, \stackrel{* *}{p}<<.001\right)$.

\begin{tabular}{|c|c|c|c|c|c|c|c|}
\hline & & $5: 6$ & $5: 7$ & $5: 8$ & $5: 9$ & $5: 10$ & $5: 5 s$ \\
\hline \multirow{2}{*}{$\begin{array}{c}\text { MultiMatch } \\
\text { similarity }\end{array}$} & $\begin{array}{l}\text { value } \\
{[0-1]}\end{array}$ & .89 & .92 & .91 & .90 & .89 & .74 \\
\hline & $t$ to $5: 5 \mathrm{~s}$ & $6.3^{* * *}$ & $9.1^{* * *}$ & $7.7^{* * *}$ & $4.1^{* *}$ & $3.7^{* *}$ & \\
\hline \multirow{4}{*}{$\begin{array}{l}\text { FuncSim } \\
\text { difference }\end{array}$} & $\begin{array}{c}\text { value } \\
{\left[{ }^{\circ} \text { v.a. }\right]} \\
\end{array}$ & 3.76 & 3.50 & 3.45 & 3.47 & 3.34 & 10.88 \\
\hline & $t$ to $5: 5 \mathrm{~s}$ & $14.9^{* * * *}$ & $15.0^{* * *}$ & $15.1^{* * * *}$ & $19.4^{* * *}$ & $17.1^{* * *}$ & \\
\hline & $\begin{array}{c}t \text { to } \\
5.93^{\circ} \text { v.a. }\end{array}$ & $7.8^{* * *}$ & $9.3^{* * *}$ & $9.6^{* * *}$ & $12.8^{* * *}$ & $11.5^{* * *}$ & \\
\hline & $\begin{array}{c}t \text { to } \\
3.06^{\circ} \text { v.a. }\end{array}$ & $2.5^{*}$ & 1.7 & 1.4 & 2.2 & 1.3 & \\
\hline
\end{tabular}




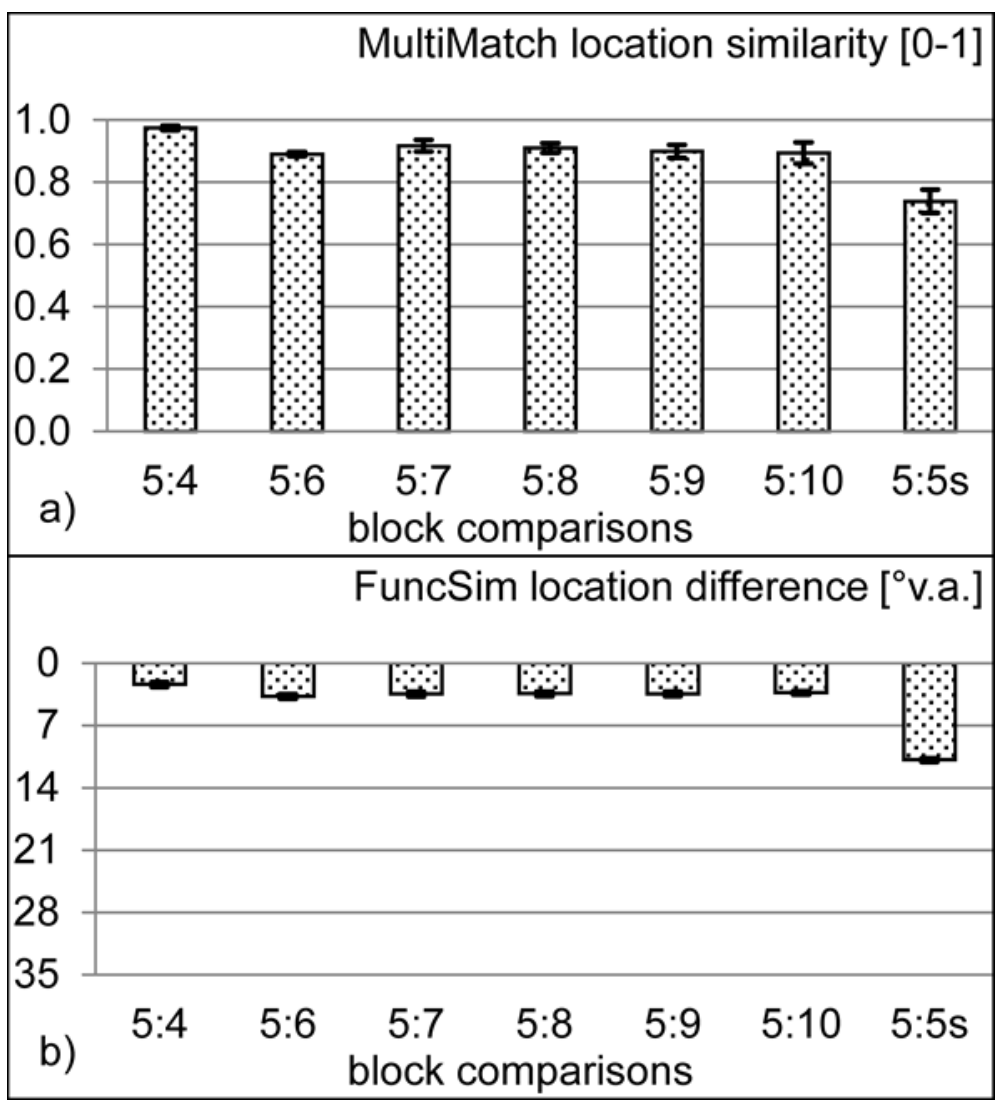

Figure 3. Mean location sequence MultiMatch similarities (a) and FuncSim differences (b) of all trials of the last visual block (block 5) to all trials of the memory blocks (blocks 6-10) as well as to 10 scrambled derivatives per block 5 trial (5s). The MultiMatch y-axis measures similarity in normalized values and ranges from the minimum possible similarity of 0 to the maximum possible similarity of 1 . The FuncSim y-axis measures difference in ${ }^{\circ} \mathrm{v}$.a. and ranges from the screen diagonal of approximately $35^{\circ} \mathrm{v} . \mathrm{a}$. to the maximum possible similarity of $0^{\circ} \mathrm{v}$.a. Error bars represent standard errors of the mean. 


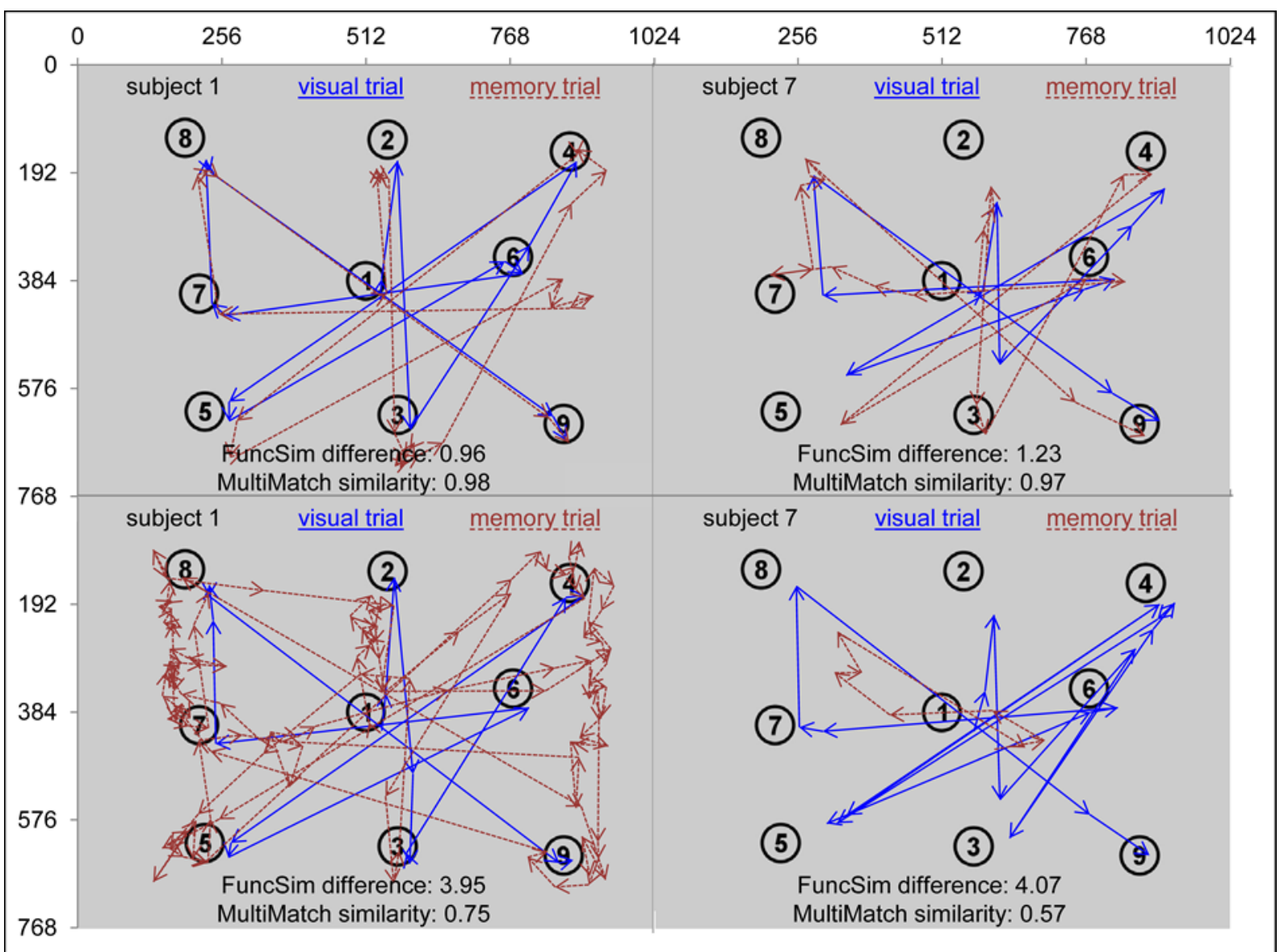

Figure 4. Example scanpaths of subject 1 (left) and subject 7 (right) during the visual (blue solid arrows) and the memory phase (red dashed arrows). The arrows represent the fixation sequence of individual trials. The number display was only visible during the visual phase. The upper two scanpath pairs represent one of the subjects' most similar comparisons, while the bottom two scanpath pairs represent one of the subjects' least similar comparisons. In the bottom left example, the memory-based scanpath contained more fixations than the visual scanpath. In the bottom right example, the memory-based scanpath contained fewer fixations than the visual scanpath. In FuncSim, both situations do not necessarily lead to decreased similarity, only if the averaged fixation locations per sub-action are dissimilar from the comparison partner. The FuncSim and MultiMatch scores for the comparison of each of the four scanpath pairs are indicated beneath each scanpath plot.

\section{Discussion}

When repeating a sensorimotor sequence for a sufficient amount of trials, participants acquire procedural memory for controlling the manual sequence, covert attention, and gaze, as indicated by target fixations (cf. Epelboim et al., 1995; Foerster \& Schneider, 2015). These guiding fixations can be used to extract visual information about the target for effector movement calculation (e.g., Prablanc, Echallier, Komilis, \& Jeannerod, 1979). However, the phenomenon of looking-at-nothing shows that empty target locations are also scanned from 
memory (Brandt \& Stark, 1997; Ferreira et al., 2008; Johansson et al., 2011, 2005, 2006; Johansson \& Johansson, 2013; Laeng \& Teodorescu, 2002; Mast \& Kosslyn, 2002; Noton \& Stark, 1971a, 1971b; Richardson \& Spivey, 2000; Spivey \& Geng, 2001). While this behavior was robustly observed in explicit memory tasks, results were mixed concerning procedural memory-based tasks, namely sensorimotor sequences (Flanagan et al., 2008; Foerster et al., 2012). More specifically, single-session investigations could not find looking-at-nothing for sensorimotor control (Flanagan et al., 2008), while a sensorimotor task that has been practiced for days was accompanied by target location fixations in complete darkness (Foerster et al., 2012). The present study aimed to reveal whether looking at remembered sensorimotor target locations can be observed in a single session observation and thus independently from sleep consolidation. Moreover, it was investigated how robustly remembered action targets are scanned by the eyes when visual information is repeatedly not available provided that task feedback is preserved.

To answer these two questions, eye movements were recorded while participants performed a manual action sequence, i.e., a computerized version of the number connection test or trail making test A (Army Individual Test Battery, 1944; Foerster \& Schneider, 2015; Reitan, 1958). In this sequential manual action task, participants had to click as fast as possible on numbered circles in ascending order. The same configuration of numbered circles had to be worked on in the same sequence for 50 trials to allow learning and accumulation of LTM. To investigate looking-at-nothing during motor control, participants thereafter had to click the sequence without visual targets on a blank screen from memory. This memory-based action phase lasted for 50 trials, so that the robustness of fixating remembered action-target locations without the availability of visual information could be studied. 


\subsection{Looking-at-nothing during single-session sensorimotor learning}

When visual information about targets was suddenly not available in the present single-session study, performance suffered in line with the previous research (cf. Flanagan et al., 2008; Foerster et al., 2012). Trial completion time increased due to more fixations.

Fixations and clicks were executed less accurately than with visual information available, indicated by larger distances to the targets' center. Probably, target locations stored in LTM come with a certain imprecision. In the visual as well as the memory-based phase, most fixations were concerned with the current target, indicated by eye-hand unit spans of about 0 . However, also subsequent targets were fixated during the visual phase (positive span), while more fixations were directed towards completed targets during the memory-based phase (negative span). Nevertheless, even when acting on remembered targets, eye movements preceded cursor movements on the way to the current target location, indicated by positive eye-hand target distance spans. Importantly, scanpaths were highly similar with and without visual information when comparing trials of the last acquisition block to trials of the first memory block. This result indicates that participants used the LTM information acquired during the visual-targets phase to shift attention and gaze sequentially to the empty target locations in the memory-based action phase. Taken together, when the motor target locations were no longer visually marked, participants scanned the empty target locations on the screen - a behavior known as looking-at-nothing (Ferreira et al., 2008; Johansson et al., 2011; Laeng \& Teodorescu, 2002; Mast \& Kosslyn, 2002; Richardson et al., 2009; Richardson \& Spivey, 2000), but less precise and less anticipatory than with visual targets available.

The observed gaze patterns are in line with Foerster et al.'s (2012) cup-stacking study. In this study, systematic scanpaths were found in the absence of visual information in complete darkness. Participants saccaded to manual target locations before the hand actually reached them in complete darkness, i.e., fixations were executed to invisible targets. In Flanagan et al. (2008), eye movements were only loosely coupled to action-target locations 
when pointing to remembered targets or manipulating a bar behind a shutter. While participants were trained for 14 days in cup-stacking before having to act in darkness (Foerster et al., 2012), participants had only short acquisition phases within a single session in the pointing and bar moving task (Flanagan et al., 2008). Therefore, the sensorimotor procedures differed in the amount of practice and thus the level of automatization as well as in whether sleep consolidation was possible. The present results indicate that looking-at-nothing can be demonstrated within a single sensorimotor session. A sufficient amount of practice rather than sleep consolidation seems to be required to elicit looking at empty target locations. Looking-at-nothing for sensorimotor control seems to depend on sufficient LTM information for controlling the sequence of attention shifts, eye movements, and manual actions.

\subsection{Progress of looking-at-nothing when visual information is repeatedly unavailable}

Looking-at-nothing during sensorimotor tasks is applied, when visual action targets are suddenly unavailable after a sufficient amount of practice with visual targets (here and in Foerster et al., 2012). However, no study so far investigated gaze behavior when participants have to perform a motor sequence repeatedly completely from memory, i.e., without the help of visual action targets. Participants might learn that eye movements can no longer be used to extract visual target information and thus abandon them. Alternatively, participants might switch from an anticipatory oculomotor target scanning to a more reflexive action completion monitoring. Finally, they may proceed or even intensify anticipatory target location scanning after being familiarized with the lack of visual information. Such an anticipatory target location scanning in the absence of visual target information could have advantages in its own as will be described in the next section.

Here, performance and gaze measures were studied over the course of 50 trials without visual targets on the screen. Most manual actions and eye movement measures changed back in direction to the values when visual targets had still been available. Manual action 
1 performance became less erroneous and faster - probably due to the reduction in fixations.

2 Fixations and clicks landed nearer to the current target's center, indicating that sequential scanning became more precise again. As auditory feedback about clicking success was still provided during the memory-based action phase, participants could use it to further refine their LTM representation of the target locations. Indeed, the consistent slightly negative value of the eye-hand unit span indicates that participants more often re-fixated a completed target location. In this way, they could check where exactly they had clicked correctly after hearing the correct tone in order to refine their spatial LTM representation. However, participants did not switch completely from an anticipatory to a reflexive gaze behavior, as indicated by the positive eye-hand time spans in later memory blocks and positive eye-hand target distance spans throughout the experiment. It seems that in addition to monitoring the success of their actions, participants also resumed to use their eyes for guiding the cursor to the current target location. Note that the performance and gaze measures in later memory-blocks might even provide a more realistic picture of how participants use their attention and gaze to solve a sensorimotor task without visual target information as the switch to the empty display is likely accompanied by a surprise effect in the early memory-based trials (cf. Foerster, 2016; Horstmann, 2015).

\subsection{Possible functions of fixating remembered action-target locations}

Why are visually available as well as remembered action-target locations sequentially scanned during sensorimotor control? When visual information is present, important features of an action target such as its orientation, surface, and size can be extracted by a fixation. These features are important to calculate the needed hand-movement parameters such as direction, force, and grip aperture (e.g., Prablanc et al., 1979; Prablanc, Pellison, \& Goodale, 1986). However, even if visual targets are not available, saccading to a target location might be functional (e.g., Ballard, Hayhoe, Pook, \& Rao, 1997; Flanagan et al., 2008) as will be 
outlined in the following. Alternatively, looking at remembered target locations in welllearned manual action sequences could just be a by-product of learning and automatization. Fixating an empty target location might facilitate declarative as well as procedural memory retrieval. Recalling target features such as size or orientation from declarative memory can help to adapt the effector movements to a specific target. There is evidence that eye movements are indeed helpful for remembering previously presented material. When recalling disappeared visual information, fixating the corresponding location helps remembering the item and rebuilding a mental image (Cabeza, Ciaramelli, Olson, \& Moscovitch, 2008; Hebb, 1968; Johansson et al., 2011; Johansson \& Johansson, 2013; Laeng \& Teodorescu, 2002; Mast \& Kosslyn, 2002; Noton \& Stark, 1971b; Olsen \& Chiew, 2014). Shifting attention and eye movements in a similar way as during encoding seems to help extracting even more and higher quality information from a memory-stored picture (Johansson, Holsanova, Johansson, Dewhurst, \& Holmqvist, 2012). Memory performance for a specific item is better when fixating the location where the item has been presented than when forced to fixate a mismatching location (Johansson \& Johansson, 2013). Eye movements are even related to memory encoding and retrieval of material encoded from verbal description only (Johansson et al., 2011, 2005, 2006), arguing that eye movements are used to connect an internal representation and a location in the external world (see also Richardson et al., 2009; Spivey \& Geng, 2001 for a similar argumentation). Especially the spatial relationship between memory items seems to be recalled easier when fixating the remembered locations (Olsen \& Chiew, 2014). Importantly, also procedural memory of required actions might be facilitated by looking at locations where these actions had been completed during prior executions. Saccading to a potential target location might work similarly as perceiving an object with a motor affordance - it activates an associated action from procedural memory (e.g., Mecklinger, Gruenewald, Weiskopf, \& Doeller, 2004; Richardson, Spivey, \& Cheung, 2001). 
1 A procedural retrieval benefit could explain why looking-at-nothing is stronger with highly-

2 practiced sensorimotor tasks (here and in Foerster et al., 2012) than with unfamiliar sensorimotor tasks (Flanagan et al., 2008). Taken together, shifting the eyes to remembered manual action target locations might help to recall target features and learned actions.

Not only memory retrieval, but also memory encoding and consolidation might be facilitated by looking-at-nothing. There is extensive evidence that rehearsal of spatial information is disturbed when eye movements have to be shifted away from the to-bememorized locations (Hale, Myerson, Rhee, Weiss, \& Abrams, 1996; Johansson \& Johansson, 2013; Lawrence, Myerson, \& Abrams, 2004; Lawrence, Myerson, Oonk, \& Abrams, 2001; Tas, Luck, \& Hollingworth, 2016). This finding has been interpreted as evidence for an eye-movement based rehearsal strategy as proposed by Baddeley (1986). Looking-at-nothing as rehearsal strategy could explain why in the present study a higher amount of fixations was concerned with completed target locations during the memory phase than during the visual phase, indicated by a slightly negative eye-hand unit span. Presumably, participants looked back at the correctly clicked locations in order to memorize the exact position for the next trial.

Moreover, it has been argued that fixating remembered action-target locations has direct benefits for hand-movement parameter calculation. Flanagan et al. (2008) argued that saccading to hand-movement target locations might facilitate the computation of the handmovement command because these sensorimotor transformations are well-learned. Similarly, Ballard and colleagues (Ballard et al., 1992, 1997) argue that the eyes might be used as an deictic pointer for the hand movements in a “do-it-where-I'm-looking” manner. The idea is that the current gaze position on a remembered target location is used as a deictic pointer for the effector movement. Neurons that are tuned to zero-disparity at the fovea code the current fixation location in external space (Ballard et al., 1997). Target locations remembered 
respective to fixation points can then be retrieved in an externally-coded coordinate and can be used to program the corresponding effector movement.

Finally, looking at remembered target locations, while performing manual action sequences without visual information available, could just be an epiphenomenon of learning and automatization. A task-adapted scanpath is learned and automatized during acquisition.

The automatized gaze routine might be applied during the memory-based manual routine even if it is no longer beneficial to scan the target locations sequentially as no visual information can be extracted (for an overview how motor routines are applied, see Ballard, 2015).

Scanning target locations sequentially is no prerequisite for LTM-based clicking, although participants mostly did so spontaneously and robustly. Whether and how looking-at-nothing is functional for motor control has to be investigated in future studies.

\section{Acknowledgements}

This research was supported by the Cluster of Excellence Cognitive Interaction Technology 'CITEC’ (EXC 277) at Bielefeld University, which is funded by the German Research Foundation (DFG).

\section{References}

Army Individual Test Battery. (1944). Manual of Directions and Scoring. War Department, Adjutant General’s Office, Washington, DC.

Baddeley, A. D. (1986). Working Memory. Oxford University Press.

Ballard, D. H. (2015). Brain computation as hierarchical abstraction. Cambridge, Massachusetts: MIT Press.

Ballard, D. H., Hayhoe, M. M., Li, F., Whitehead, S. D., Frisby, J. P., Taylor, J. G., \& Fisher, R. B. (1992). Hand-eye coordination during sequential tasks. Philosophical Transactions: Biological Sciences, 337, 331-339. 
1 Ballard, D. H., Hayhoe, M. M., Pook, P. K., \& Rao, R. P. N. (1997). Deictic codes for the embodiment of cognition. The Behavioral and Brain Sciences, 20, 723-742.

Brandt, S. A., \& Stark, L. W. (1997). Spontaneous eye movements during visual imagery reflect the content of the visual scene. Journal of Cognitive Neuroscience, 9, 27-38.

Butsch, R. L. C. (1920). Eye movements and the eye-hand span in typewriting. Journal of Educational Psychology, 23, 104-121.

Cabeza, R., Ciaramelli, E., Olson, I. R., \& Moscovitch, M. (2008). The parietal cortex and episodic memory: An attentional account. Nature Reviews Neuroscience, 9, 613-625. doi:10.1038/nrn2459

Deubel, H., \& Schneider, W. X. (1996). Saccade target selection and object recognition: Evidence for a common attentional mechanism. Vision Research, 36, 1827-1837. doi:10.1016/0042-6989(95)00294-4

Dewhurst, R., Nyström, M., Jarodzka, H., Foulsham, T., Johansson, R., \& Holmqvist, K. (2012). It depends on how you look at it: Scanpath comparison in multiple dimensions with MultiMatch, a vector-based approach. Behavior Research Methods, 44, 1079-1100. doi:10.3758/s13428-012-0212-2

Epelboim, J. L., Steinman, R. M., Kowler, E., Edwards, M., Pizlo, Z., Erkelens, C. J., \& Collewijn, H. (1995). The function of visual search and memory in sequential looking tasks. Vision Research, 35, 3401-3422.

Ferreira, F., Apel, J., \& Henderson, J. M. (2008). Taking a new look at looking at nothing. Trends in Cognitive Sciences, 12, 405-410. doi:10.1016/j.tics.2008.07.007

Flanagan, J. R., Terao, Y., \& Johansson, R. S. (2008). Gaze behavior when reaching to remembered targets. Journal of Neurophysiology, 100, 1533-1543. doi:10.1152/jn.90518.2008.

Foerster, R. M. (2016). Task-irrelevant expectation violations in sequential manual actions: Evidence for a “check-after-surprise” mode of visual attention and eye-hand decoupling. 
Frontiers in Psychology, 7, 1-12. doi:10.3389/fpsyg.2016.01845

Foerster, R. M., Carbone, E., Koesling, H., \& Schneider, W. X. (2011). Saccadic eye movements in a high-speed bimanual stacking task: Changes of attentional control during learning and automatization. Journal of Vision, 11(7):9, 1-16. doi:10.1167/11.7.9

Foerster, R. M., Carbone, E., Koesling, H., \& Schneider, W. X. (2012). Saccadic eye movements in the dark while performing an automatized sequential high-speed sensorimotor task. Journal of Vision, 12(2):8, 1-15. doi:10.1167/12.2.8

Foerster, R. M., \& Schneider, W. X. (2013a). FuncSim Toolbox for Matlab: Computation of eyetracking scanpath similarity. Bielefeld University. doi:10.4119/unibi/citec.2013.7

Foerster, R. M., \& Schneider, W. X. (2013b). Functionally sequenced scanpath similarity method (FuncSim): Comparing and evaluating scanpath similarity based on a task's inherent sequence of functional. Journal of Eye Movement Research, 6, 1-22.

Foerster, R. M., \& Schneider, W. X. (2015). Expectation violations in sensorimotor sequences: shifting from LTM-based attentional selection to visual search. Annals of the New York Academy of Sciences, 1339, 45-59. doi:10.1111/nyas.12729

Furneaux, S., \& Land, M. F. (1999). The effects of skill on the eye-hand span during musical sight-reading. Proceedings of the Royal Society B Biological Sciences, 266, 2435-2440. doi:10.1098/rspb.1999.0943

Hale, S., Myerson, J., Rhee, S. H., Weiss, C. S., \& Abrams, R. A. (1996). Selective interference with the maintenance of location information in working memory. Neuropsychology, 10, 228-240.

Hebb, D. O. (1968). Concerning imagery. Psychological Review, 75, 466-477.

Horstmann, G. (2015). The surprise-attention link: a review. Annals of the New York Academy of Sciences, 1-10. doi:10.1111/nyas.12679

Jarodzka, H., Holmqvist, K., \& Nyström, M. (2010). A vector-based, multidimensional scanpath similarity measure. Proceedings of the 2010 Symposium on Eye-Tracking 
Research \& Applications, 211-218. doi:10.1145/1743666.1743718

Johansson, R., Holsanova, J., Dewhurst, R., \& Holmqvist, K. (2011). Eye movements during scene recollection have a functional role, but they are not reinstatements of those produced during encoding. Journal of Experimental Psychology: Human Perception and Performance, 38, 1289-1314. doi:10.1037/a0026585

Johansson, R., Holsanova, J., \& Holmqvist, K. (2005). What do eye movements reveal about mental imagery? Evidence from visual and verbal elicitations. In Proceedings of the 27th Cognitive Science Conference (pp. 49-56). Stresa, Italy: Erlbaum.

Johansson, R., Holsanova, J., \& Holmqvist, K. (2006). Pictures and spoken descriptions elicit similar eye movements during mental imagery, both in light and in complete darkness. Cognitive Science, 30, 1053-1079. doi:10.1207/s15516709cog0000_86

Johansson, R., Holsanova, J., Johansson, M., Dewhurst, R., \& Holmqvist, K. (2012). Eye movements play an active role when visuospatial information is recalled from memory. In Vision Sience Society (p. 1). Naples, FL,USA: Journal of Vision.

Johansson, R., \& Johansson, M. (2013). Look here, eye movements play a functional role in memory retrieval. Psychological Science, 1-7. doi:10.1177/0956797613498260

Laeng, B., \& Teodorescu, D.-S. (2002). Eye scanpaths during visual imagery reenact those of perception of the same visual scene. Cognitive Science, 26, 207-231. doi:10.1207/s15516709cog2602_3

Lawrence, B. M., Myerson, J., \& Abrams, R. A. (2004). Interference with spatial working memory: An eye movement is more than a shift of attention. Psychonomic Bulletin and Review, 11, 488-494.

Lawrence, B. M., Myerson, J., Oonk, H. M., \& Abrams, R. A. (2001). The effects of eye and limb movements on working memory. Memory, 9, 433-444. doi:10.1080/09658210143000047

Mast, F. W., \& Kosslyn, S. M. (2002). Eye movements during visual mental imagery. Trends 
in Cognitive Sciences, 6, 271-272.

Mecklinger, A., Gruenewald, C., Weiskopf, N., \& Doeller, C. F. (2004). Motor affordance and its role for visual working memory: Evidence from fMRI studies. Experimental Psychology, 51, 258-269. doi:10.1027/1618-3169.51.4.258

Noton, D., \& Stark, L. (1971a). Scanpaths in eye movements during pattern perception. Science, 171, 308-311.

Noton, D., \& Stark, L. (1971b). Scanpaths in saccadic eye movements while viewing and recognizing patterns. Vision Research, 11, 929-942.

Olsen, R., \& Chiew, M. (2014). The relationship between delay period eye movements and visuospatial memory. Journal of Vision, 14(1), 1-11. doi:10.1167/14.1.8.doi

Prablanc, C., Echallier, J. F., Komilis, E., \& Jeannerod, M. (1979). Optimal response of eye and hand motor systems in pointing at a visual target. Biological Cybernetics, 35, 113124.

Prablanc, C., Pellison, D., \& Goodale, M. A. (1986). Visual control of reaching movements without vision of the limb. Experimental Brain Research, 62, 293-302.

Reitan, R. M. (1958). Validity of the trail making test as an indicator of organic brain damage. Perceptual and Motor Skills, 8, 271-276.

Richardson, D. C., Altmann, G. T. M., Spivey, M. J., \& Hoover, M. A. (2009). Much ado about eye movements to nothing: a response to Ferreira et al.: Taking a new look at looking at nothing. Trends in Cognitive Sciences, 13, 235-236. doi:10.1016/j.tics.2009.02.006

Richardson, D. C., \& Spivey, M. J. (2000). Representation, space and Hollywood Squares: looking at things that aren’t there anymore. Cognition, 76, 269-295. doi:10.1016/S00100277(00)00084-6

Richardson, D. C., Spivey, M. J., \& Cheung, J. (2001). Motor representations in memory and mental models: Embodiment in cognition. In Proceedings of the Twenty-third Annual 
Meeting of the Cognitive Science Society (pp. 867-872). Erlbaum: Mawhah, NJ.

Sailer, U., Eggert, T., Ditterich, J., \& Straube, A. (2000). Spatial and temporal aspects of eyehand coordination across different tasks. Experimental Brain Research, 134, 163-173. doi:10.1007/s002210000457

Spivey, M. J., \& Geng, J. J. (2001). Oculomotor mechanisms activated by imagery and memory: Eye movements to absent objects. Psychological Research, 65, 235-241.

Stickgold, R. (2005). Sleep-dependent memory consolidation. Nature, 437, 1272-1278. doi:10.1038/nature04286

Tas, A. C., Luck, S. J., \& Hollingworth, A. (2016). The relationship between visual attention and visual working memory encoding: A dissociation between covert and overt orienting. Journal of Experimental Psychology: Human Perception and Performance, 42, 1121-1138. doi:10.1037/xhp0000212

Walker, M. P., Brakefield, T., Morgan, A., Hobson, J. A., \& Stickgold, R. (2002). Practice with sleep makes perfect: Sleep-dependent motor skill learning. Neuron, 35, 205-211. doi:10.1016/S0896-6273(02)00746-8

8 Supplementary material Supplementary material can be found on the website of Vision Research. The file named 'FigureS1' depicts example scanpaths based on the 1,000 Hz raw data samples of subject 1 (left) and subject 7 (right) during the visual (blue) and the memory phase (red). The number display was only visible during the visual phase. The upper two scanpath pairs represent one of the subjects' most similar comparisons, while the bottom two scanpath pairs represent one of the subjects' least similar comparisons. The corresponding fixation sequences and their FuncSim and MultiMatch comparison scores can be seen in Figure 4. The files with the prefix 'slowmo' contain slow-motion eye (moving diamond and broken trace) and cursor (moving dot and solid trace) movements $(100 \mathrm{~Hz})$ of the trial pairs presented in Figure 4 and FigureS1 
1 (visual trials in blue and memory trials in red). The number display was only visible during

2 the visual phase. The correspondence to Figure 4 and FigureS1 is indicated by the file name

3 (subject[No] and low vs. high for type of similarity). In the files with the suffix 'aligned', the

4 movements were aligned per click action to facilitate the comparison of the eye-cursor

5 dynamics in the visual and the memory phase when acting on the same target. 